\title{
ORIGINAL
}

\section{EVOLUCIÓN DEL CONSUMO DE FÁRMACOS ANTIDEPRESIVOS EN LAS ÁREAS SANITARIAS DE LA RIOJA Y ZAMORA DURANTE EL PERIODO 1997-2001}

Carlos Sainz de Rozas Aparicio (1), María Teresa Ruiz Clavijo Díez (2) y Alfonso Díaz Madero (3)

(1) Servicio de Farmacia. Complejo Hospitalario San Millán San Pedro. Logroño.

(2) Gerencia de Atención Primaria de Logroño

(3) Gerencia de Atención Primaria de Zamora.

\section{RESUMEN}

Fundamento: Debido al gran aumento del consumo de antidepresivos, y al cambio del perfil de prescripción dentro del grupo, el objetivo del estudio es describir la evolución del consumo extrahosde La Rioja y Zamora durante el periodo 1997-2001 y valorar el impacto de los nuevos fármacos.

Métodos: Se ha estudiado el consumo de fármacos incluidos en el subgrupo terapéutico N06A de la Clasificación Anatómica Terapéutica Química. Los datos de consumo de cada especialidad se han obtenido de los programas informáticos de gestión de farmacia (SIFAR) y se han expresado para cada principio activo en Dosis Habitante Día (DHD).

Resultados: En el periodo de estudio se ha producido un incremento del consumo total de antidepresivos en las dos áreas (Zamora $55 \%$ y La Rioja 93\%), debido fundamentalmente al incremento de los inhibidores selectivos de la recaptación de serotonina (ISRS) (Zamora 80\% y La Rioja 143\%) y de los nuevos antidepresivos heterocíclicos (Zamora 82\% y La Rioja 78\%).

Conclusiones: El incremento en la utilización de antidepresivos va acompañado de un cambio en el perfil de prescripción: hay un e los ISRS y algunos heterocíclicos (venlafaxina, trazodona, reboxetina), disminuyendo la utilización de los antidepresivos tricíclicos y de los inhibidores de la monoaminooxidasa.

Palabras clave: Agentes antidepresivos. Depresión. Utilización de medicamentos.

Correspondencia:

Carlos Sainz Rozas Aparicio

Servicio de Farmacia

Complejo Hospitalario San Millán San Pedro.

Correo electrónico: csainz@hsm.seris.es

\section{ABSTRACT}

\section{Evolution of the Utilization} of Antidepressant Drugs in the Rioja and Zamora Health Districts Throughout the 1997-2001 Period

Background: Due to the high increase in the utilization of antidepressant drugs and the change in the prescription profile within this group, this study is aimed at discovering the evolution of the utilization of antidepressant drugs (NO6A subgroup) outside of the hospital setting in the Rioja and Zamora health districts throughout the 1997-2001 period and to evaluate the impact of the new drugs.

Methods: A study was made of the utilization of drugs included in the NO6A treatment subgroup under the Anatomical Therapeutic Chemical (ATC) classification. The data on the utilization of each specialty was taken from the pharmacy management (SIFAR) software applications and has been stated for each active ingredient in Daily Dose per Inhabitant (DDI)

Results: In the period under study, there was an increase in the total utilization of antidepressants in the two areas (Zamora 55\% and Rioja 93\%) due mainly to the increase in selective serotinin reuptake inhibitors (SSRI) (Zamora 80\% and Rioja 143\%) and in the new heterocyclic antidepressants (Zamora 82\% and Rioja 78\%).

Conclusions: There has been an increase in the utilization of antidepressants, all of which has taken place in conjunction with a change in the prescription profile. There is a clear shift toward the utilization of the SSRI's and some heterocyclic antidepressants (venlafaxine, trazodone, reboxetine), the utilization of tricyclic antidepressants and IMAO's having decreased.

Key words: Antidepressive Agents. Depression. Pharmacoepidemiology. Drug utilization. 


\section{INTRODUCCIÓN}

Durante los últimos años, numerosos estudios clínicos y epidemiológicos, señalan que la depresión es un trastorno muy frecuente y en progresivo aumento. La relevancia de su frecuencia cobra un significado mayor si se tiene en cuenta que este trastorno genera una discapacidad funcional importante, superior incluso a la mayoría de las enfermedades crónicas ${ }^{1}$.

Aunque las cifras de incidencia y prevalencia halladas en los diferentes estudios realizados varían en función de la metodología empleada, se estima que la prevalencia anual de la depresión en la población general se sitúa entre el 4-6\% $\%^{1,2}$. Extrapolando estos datos, el Comité para la prevención y tratamiento de la depresión estima que tasa de prevalencia de depresión en España oscila entre 1.200 .000 y 1.500 .000 , aproximadamente el $10 \%$ de los adultos ${ }^{1}$.

Los trastornos depresivos son uno de los motivos de consulta más frecuentes en Atención Primaria ${ }^{3}$. En un estudio realizado por la OMS en centros de Atención Primaria de 14 países se constató que hasta el 10\% de los pacientes que acuden a consulta de Atención Primaria pueden padecer depresión ${ }^{4-8}$. Vázquez Barquero, en un estudio realizado sobre primeras consultas en Atención Primaria en nuestro medio encuentra tasas de prevalencia ponderada de depresión de 4,5\% para los hombres y del 7,8\% para las mujeres ${ }^{9}$.

La depresión es un problema importante en la clínica diaria de los médicos de Atención Primaria. Pero además, la depresión es una de las principales cargas sanitarias como consecuencia del impacto social que provoca, del elevado precio del tratamiento, el alto índice de suicidios ${ }^{1-4}$, las altas tasas de cronicidad $(15-20 \%)^{10,11}$, y el incremento de la mortalidad debido a su asociación con enfermedades crónicas.

El tratamiento de la depresión moderada y grave consiste en administrar fármacos anti- depresivos a dosis plenas con una adecuada duración del mismo y junto con psicoterapia $^{2}$. Según lo expuesto, el consumo de estos fármacos debería ser elevado. Sin embargo un estudio realizado en nuestro país sobre el consumo de antidepresivos a través de receta sobre el periodo 1985-1994 muestra que a pesar de que el consumo se ha triplicado en los últimos 10 años, éste se encuentra muy por debajo de lo que correspondería ${ }^{12}$.

Lo anteriormente expuesto unido a la comercialización de nuevos fármacos sin los efectos secundarios de los antidepresivos más clásicos, y el hecho de existir un estudio de estas características en el Área de Zamora desde 1996-199913, nos ha llevado a realizar un estudio cuyo objetivo es describir la evolución del consumo extrahospitalario de antidepresivos en las áreas de salud de La Rioja y Zamora desde 1997 al 2001, analizar las diferencias del consumo y valorar el impacto de los nuevos fármacos en las dos áreas.

\section{MATERIAL Y METODOS}

Se ha realizado un estudio descriptivo y retrospectivo del consumo de medicamentos incluidos en el subgrupo terapéutico N06A ${ }^{14}$ (antidepresivos), en el ámbito extrahospitalario de las Áreas de Salud de La Rioja y Zamora, durante el periodo 1997-2001.

Los principios activos pertenecientes a este subgrupo se han clasificado siguiendo criterios de estructura química y acción farmacológica $^{15}$

a) Antidepresivos tricíclicos

b) Inhibidores selectivos de la recaptación de serotonina

c) Antidepresivos heterocíclicos

d) Inhibidores de la monoaminooxidasa 
No se incluyeron las asociaciones de antidepresivos con otros fármacos, ya que quedaron excluidos de la financiación pública por el Real Decreto 1663/1998 de 24 de Julio $^{16}$ y desde entonces no se dispone de datos de consumo a través de receta de Seguridad Social. Tampoco se incluye el litio, aunque se utilice en el tratamiento de las depresiones bipolares, ya que es un regulador del humor.

Los datos de consumo de cada especialidad farmacéutica se han obtenido de los sistemas informáticos de gestión de farmacia de la Gerencia de Atención Primaria de La Rioja «RIOJAFAR» y de la Gerencia de Atención Primaria de Zamora «SIFAR», que contienen información sobre el número de especialidades farmacéuticas dispensadas con recetas de la Seguridad Social por las Oficinas de Farmacia de las dos áreas ${ }^{17}$. Asumimos que el consumo obtenido a través de estas fuentes es una aproximación aceptable del consumo real, ya que el $98 \%$ de la población española está incluida en el sistema de Seguridad Social ${ }^{12}$.

Los datos de consumo se han expresado para cada principio activo en dosis habitante y día (DHD), que corresponde a la dosis diaria definida (DDD) por 1.000 habitantes/día. La DDD es una unidad técnica de medida y de comparación que se define como la dosis media diaria de mantenimiento en adultos de un medicamento cuando se utiliza en su principal indicación ${ }^{18}$.

Para este estudio se han utilizado las DDD establecidas en el Nomenclator Digitalis ${ }^{19}$, por considerarlo más completo y actualizado, ya que constan todos los fármacos comercializados en España con sus DDD y el origen o autor de estas ${ }^{13}$. Para todas las DDD figura como origen la Clasificación Anatómica Terapéutica Química, excepto para amineptina, oxitriptan, y trazodona que aparece como origen o autor el Insalud. Para fenelzina se utilizó la DDD establecida por el Nordic Council on Medicines ${ }^{20}$.
Para el cálculo de la DHD se utilizó la siguiente fórmula ${ }^{17}$ :

$$
\begin{gathered}
\text { DHD= Núm de DDD } \times 1.000 \\
\text { hab/Población } \times 365
\end{gathered}
$$

Siendo el $\mathrm{N}^{\circ}$ DDD $=\mathrm{N}^{\mathrm{o}}$ de envases dispensados anualmente $\times$ Núm de unidades de cada envase $\times$ mg de principio activo por forma farmacéutica / DDD estándar en mg.

La población del denominador es la asignada por la Subdirección General de Atención Primaria del Insalud para los contratos de gestión para cada año y áreas estudiadas, según los registros de la tarjeta individual sanitaria..

Los criterios de evaluación que se han utilizado son ${ }^{13}$ :

1. DHD de cada fármaco y de cada área sanitaria

2. Porcentaje de incremento del 2001 respecto a 1997:

[(DHD2001-DHD1997)/ DHD 1997] × 100

\section{RESULTADOS}

Se observa un crecimiento progresivo en el consumo total de antidepresivos en las dos áreas, siendo el incremento del consumo en año 2001 respecto a 1997 superior en La Rioja $93 \%$ frente al 55\% en Zamora (figura 1). Sin embargo, el consumo total en DHD en cada una de las áreas del periodo de estudio es mayor en Zamora que en La Rioja (1997: 22,10 y 16,08 ; $1998: 24,67$ y 19,14 ; 1999: 27,86 у 20,$56 ; 2000: 31,40$ у 25,37 ; 2001: 34,15 y 31,01$)$.

La evolución del consumo de cada uno de los fármacos de los distintos grupos se expone en las tablas 1 a 5. La evolución de los antidepresivos más consumidos en las dos áreas se representan en las figuras 1 y 2 . En las figuras 3 y 4 se representa el porcentaje 
Figura 1

Evolución de los antidepresivos más consumidos en La Rioja

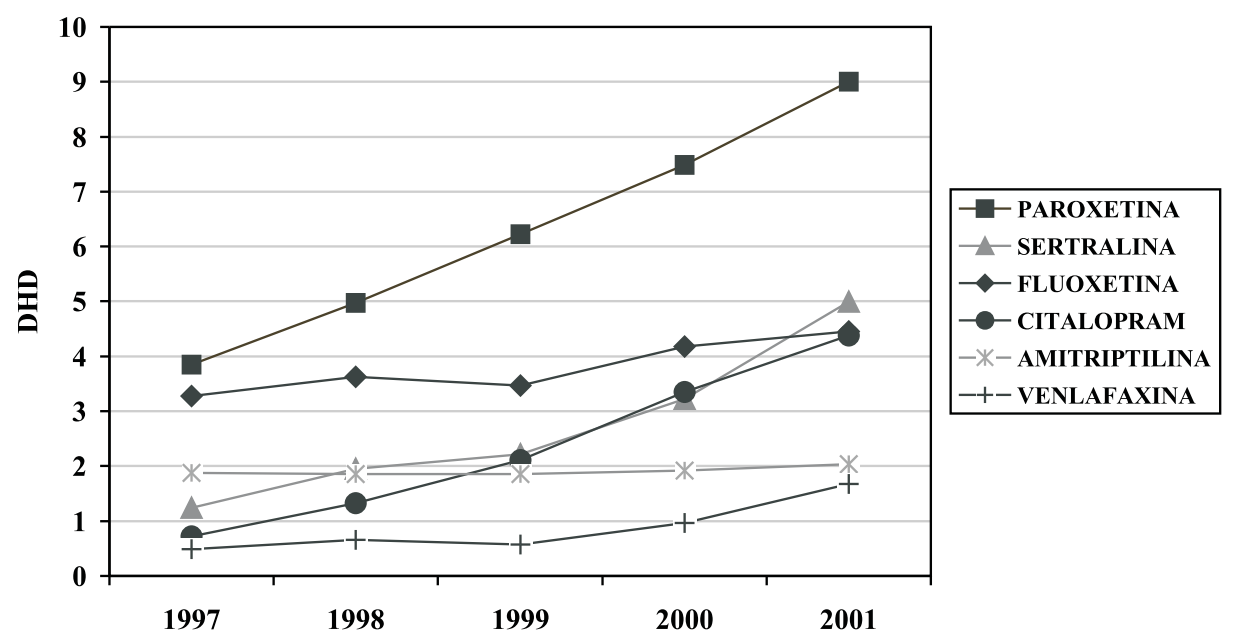

de utilización de cada subgrupo sobre el total del consumo de antidepresivos en cada área sanitaria.

en DHD es mayor en La Rioja que en Zamora $(23,31$ frente a 22,40). Citalopran, sertralina y paroxetina experimentan un importante crecimiento en las dos áreas, fluoxetina crece ligeramente y fluvoxamina disminuye un $3 \%$ en La Rioja frente al $17 \%$ de incremento que experimenta en Zamora.

El consumo de antidepresivos heterocíclicos aumenta en las dos áreas, aunque la utilización es mayor en el área de Zamora que en La Rioja. En el año 2001 la DHD en Zamora es de 7,21 frente a 4,12 en La Rioja (tabla 3).

Los principios activos responsables de este aumento son: venlafaxina con un incremento del $218 \%$ y $240 \%$ respectivamente, trazodona y reboxetina.

Presentan un comportamiento diferente la maprotilina que desciende un $48 \%$ en Zamora y $7 \%$ en La Rioja y la mianserina que disminuye su consumo en La Rioja (36\%) y aumenta ligeramente en Zamora (9\%) considerando el periodo de estudio.

Finalmente, los IMAO además de presentar un consumo irrelevante en la dos áreas, en el periodo de estudio se produce un importante descenso (tabla 4). pectivamente) (tabla 2). A diferencia de lo que ocurre en el grupo anterior, el consumo 
EVOLUCIÓN DEL CONSUMO DE FÁRMACOS ANTIDEPRESIVOS EN LAS ÁREAS SANITARIAS DE LA RIOJA Y ZAMORA...

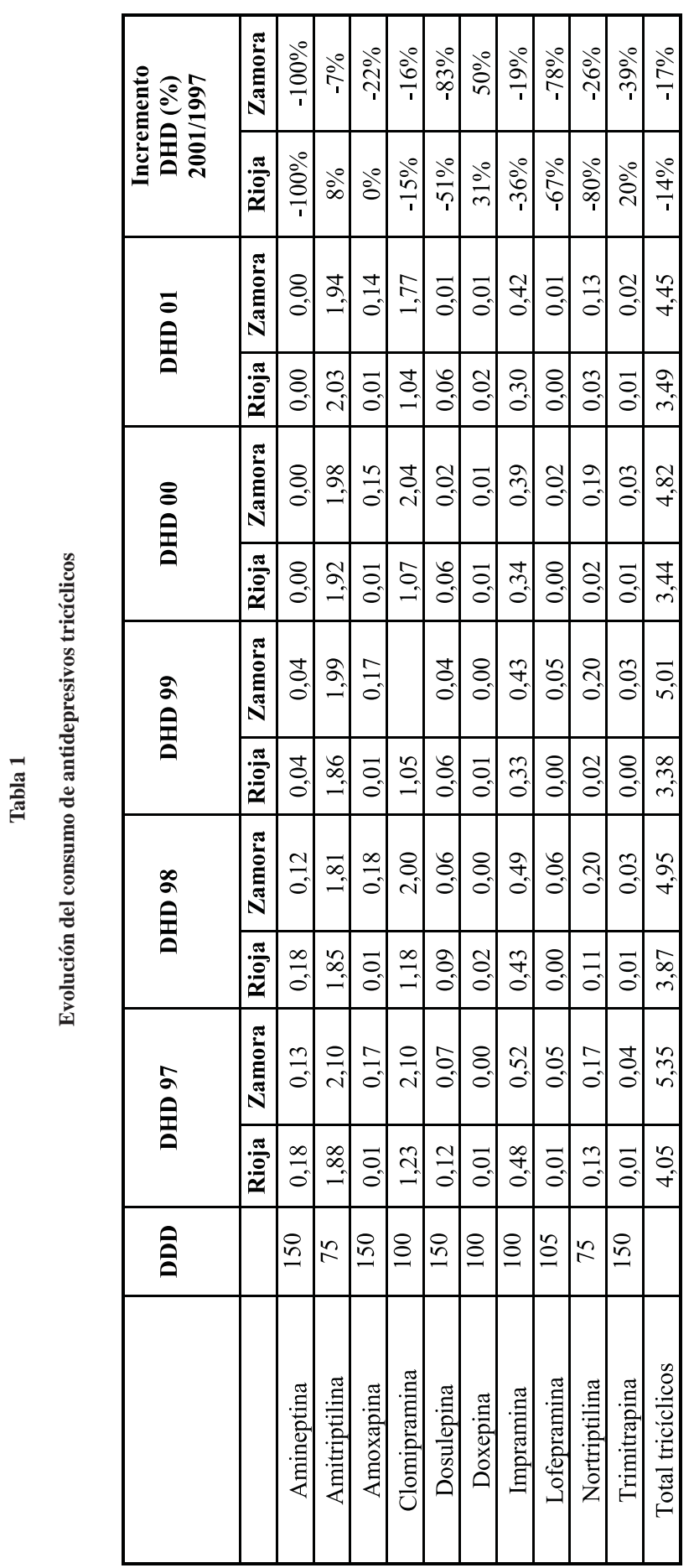

Rev Esp Salud Pública 2004, Vol. 78, N. ${ }^{\circ} 5$ 


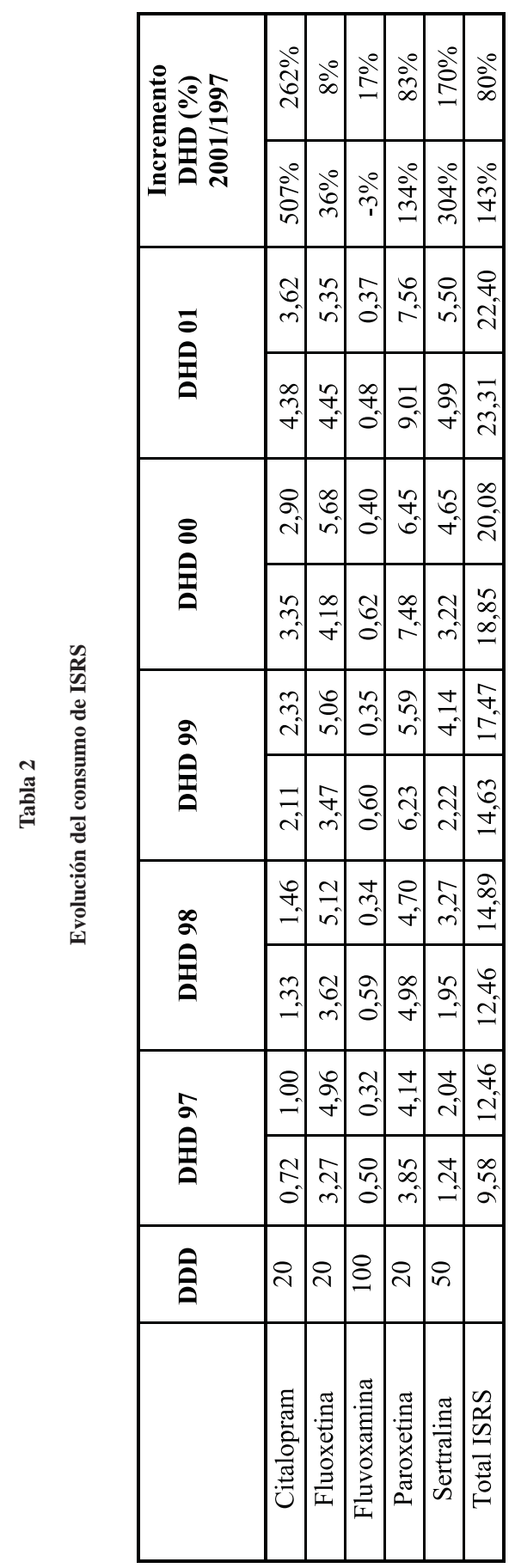


EVOLUCIÓN DEL CONSUMO DE FÁRMACOS ANTIDEPRESIVOS EN LAS ÁREAS SANITARIAS DE LA RIOJA Y ZAMORA...

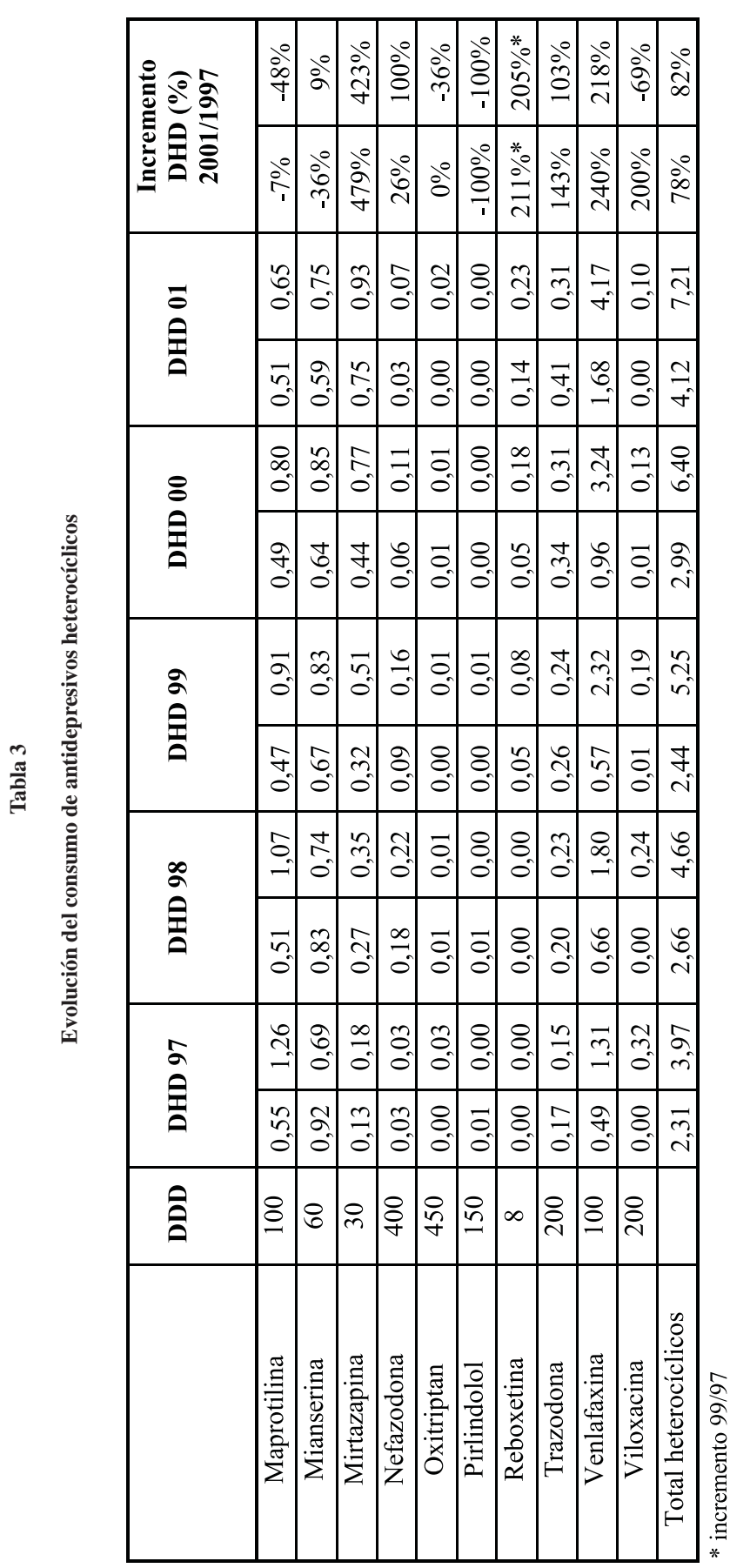

Rev Esp Salud Pública 2004, Vol. 78, N. ${ }^{\circ} 5$ 


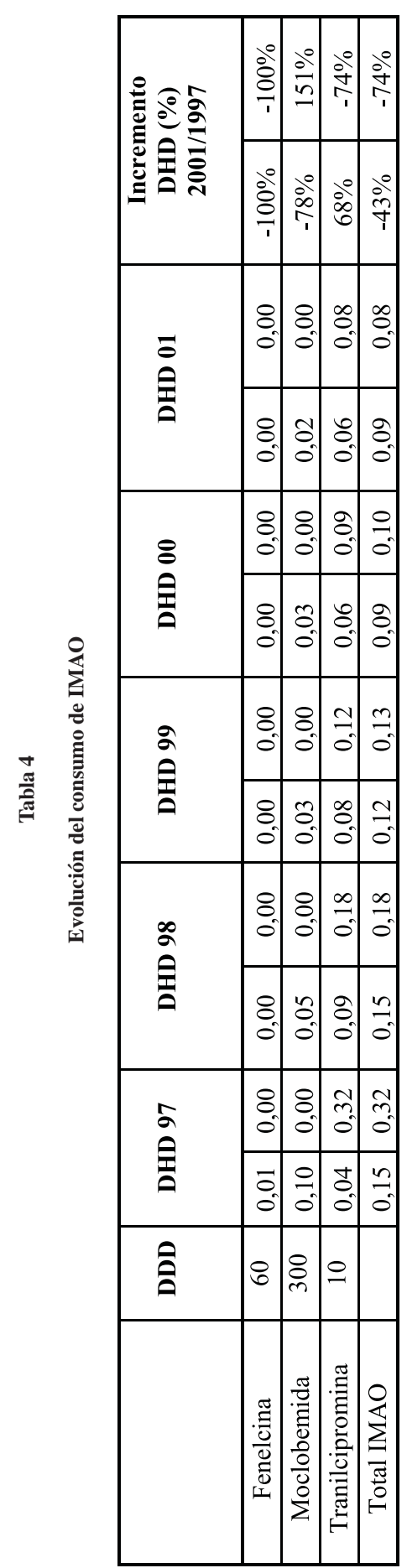


EVOLUCIÓN DEL CONSUMO DE FÁRMACOS ANTIDEPRESIVOS EN LAS ÁREAS SANITARIAS DE LA RIOJA Y ZAMORA..

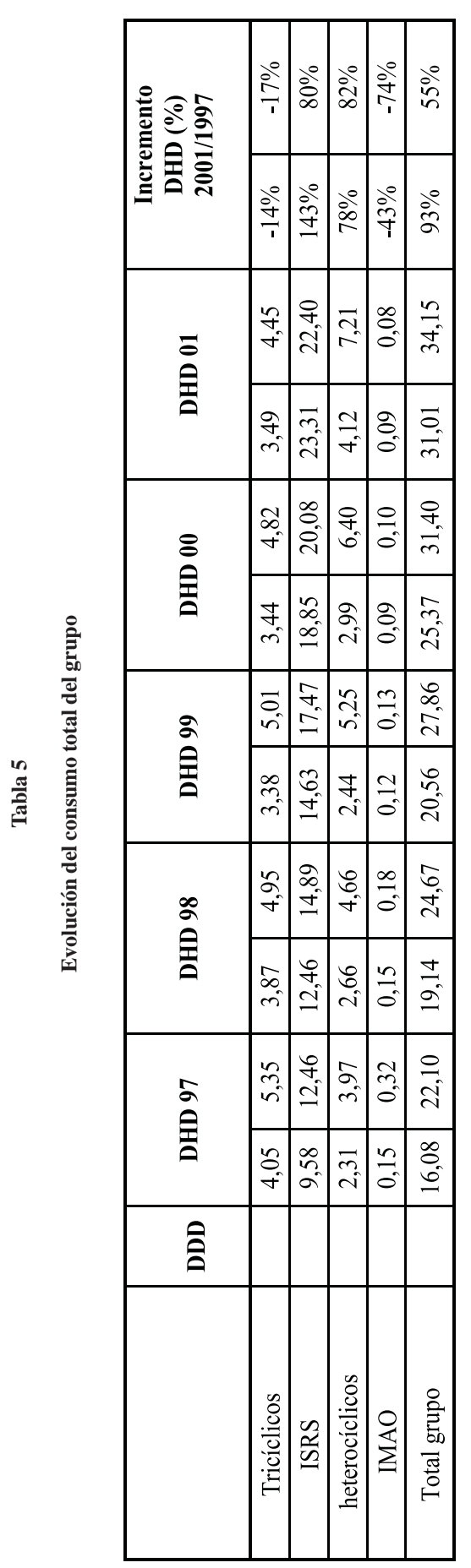

Rev Esp Salud Pública 2004, Vol. 78, N. ${ }^{\circ} 5$ 
Figura 2

Evolución de los antidepresivos más consumidos en Zamora

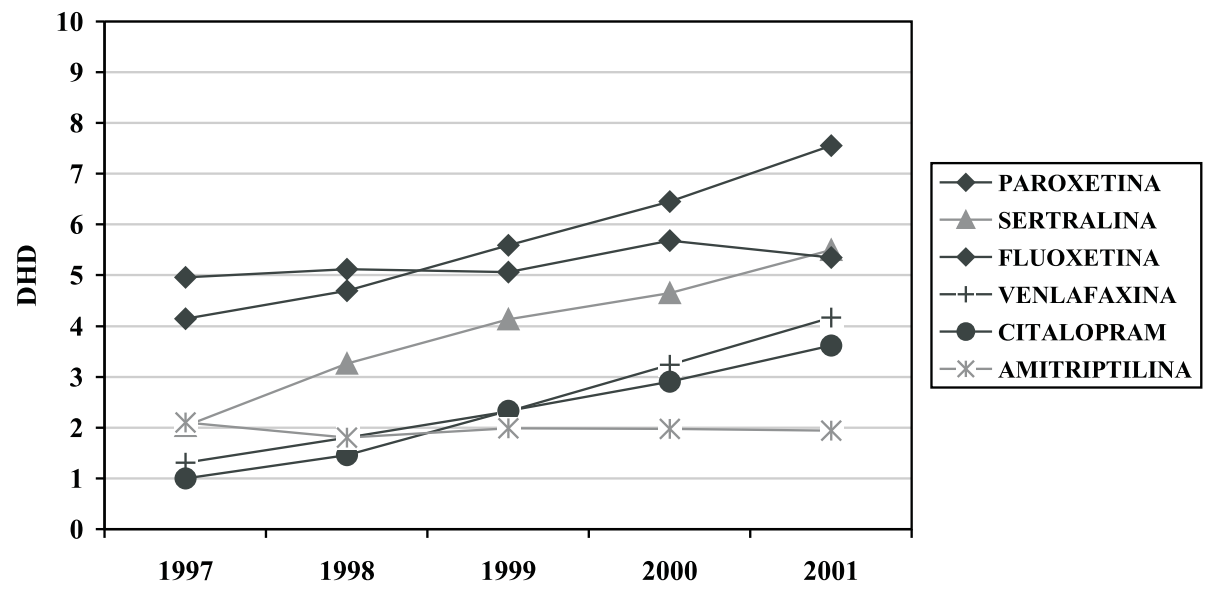

Figura 3

Porcentaje de utilización de cada subgrupo sobre el total del consumo de antidepresivos en La Rioja

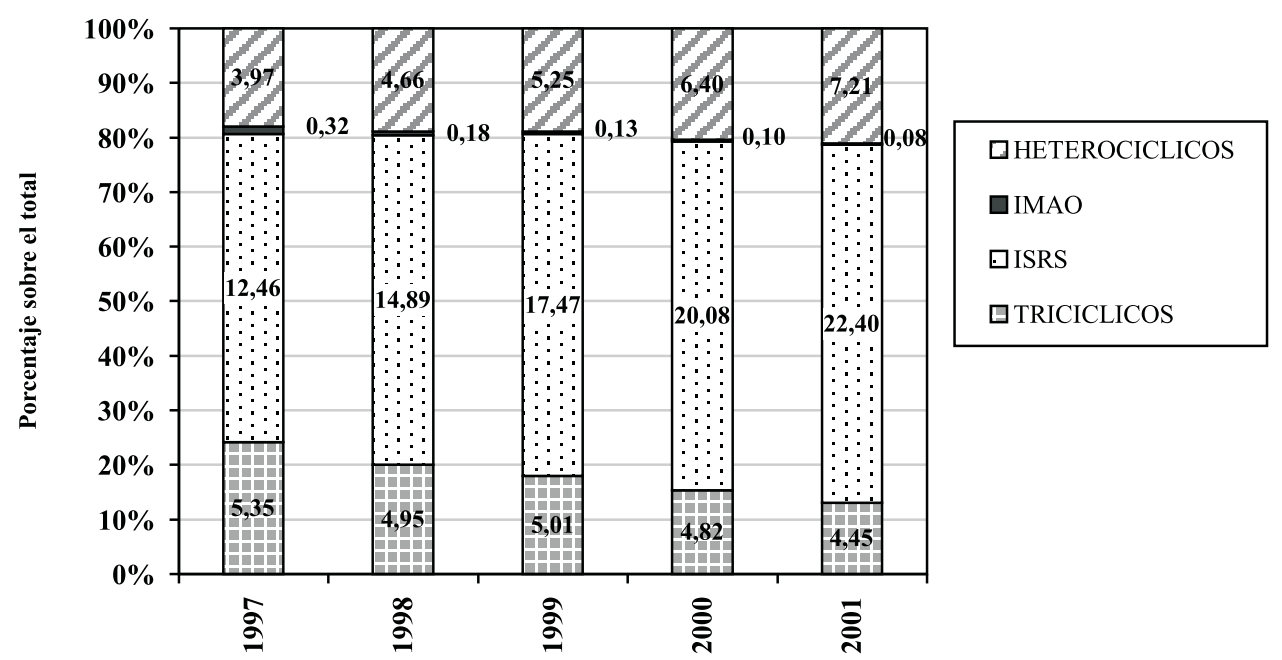

\section{DISCUSIÓN}

Antes de analizar e interpretar los resultados obtenidos, hay que tener en cuenta una serie de limitaciones ya referenciadas en estudios similares13: Sólo se incluye el consumo extrahospitalario de antidepresivos a través de receta de la Seguridad Social, sin que se conozca la dispensación sin receta (aunque esta debería ser nula ya que son medicamentos que precisan receta para su dispensación), ni las prescripciones en el ámbito privado o por otras entidades gestoras (Muface, Isfas,...). Sin embargo, consideramos que los envases de antidepresivos 
Figura 4

Porcentaje de utilización de cada subgrupo sobre el total del consumo de antidepresivos

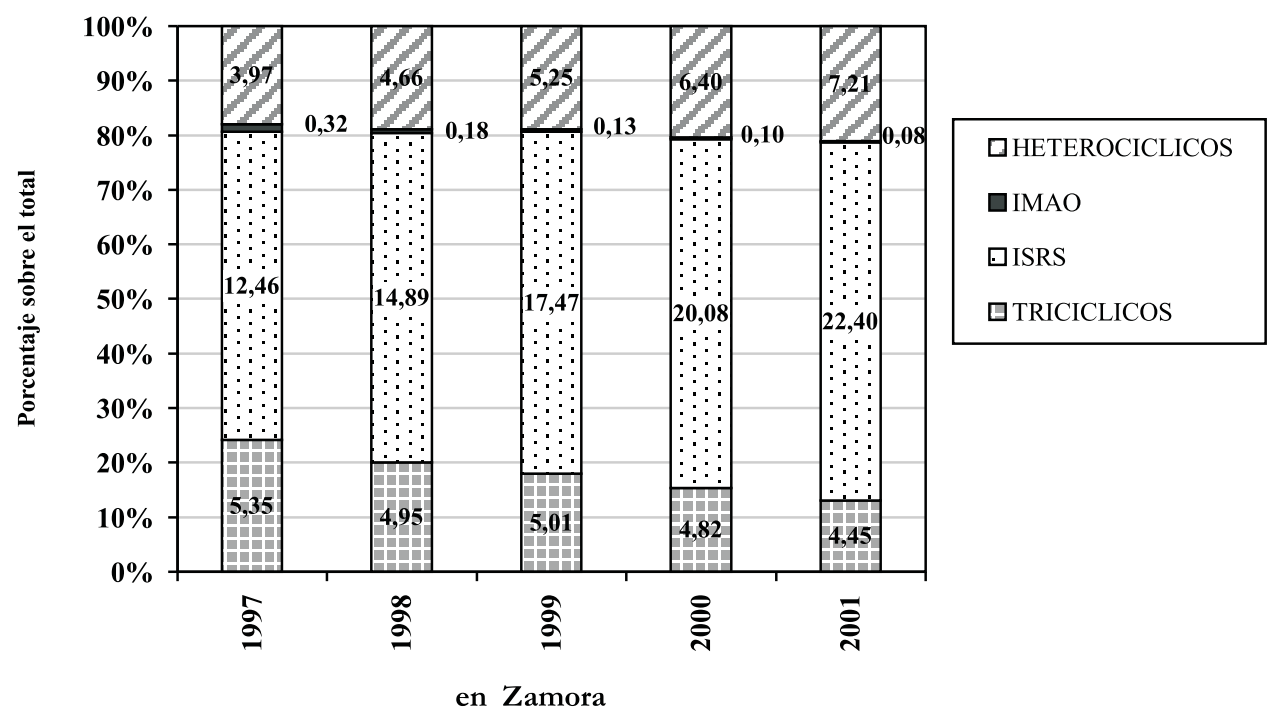

sin receta de la seguridad social representan un porcentaje muy pequeño y que, dada la cobertura prácticamente universal del sistema público, es esperable que las diferencias entre lo hallado y lo real sean mínimas.

Aunque estos fármacos mayoritariamente se prescriben para tratar la depresión, no podemos estimar la prevalencia en cada área a partir de los resultados obtenidos, ya que se desconoce la indicación real para la que fueron recetados (depresión, pánico, bulimia, diversos trastornos de ansiedad, fibromialgia, colon irritable, síndrome de fatiga crónica, etc).

Disponemos de datos de dispensación a través de la oficina de farmacia, pero ello no quiere decir que los fármacos sean consumidos o administrados en su totalidad (incumplimiento terapéutico).

Las dosis diarias definidas (DDD) utilizadas pueden ser diferentes según la indicación para la que se empleen y se considera una DDD por fármaco. Normalmente, las
DDD de ISRS son ajustadas a la práctica clínica, no así las de los tricíclicos y heterocíclicos, ya que sus DDD son inferiores a las terapéuticas.

En general debemos reconocer el valor de la DDD como unidad de medida y comparación entre ámbitos distintos, así como de marcador de la evolución de la utilización de fármacos, pero también recomendamos cautela en posteriores análisis y aplicaciones, entre los que podemos citar el cálculo de la prevalencia. Es una extrapolación arriesgada, ya que los antidepresivos tienen indicaciones psiquiátricas y no psiquiátricas.

Hechas estas consideraciones, que asumimos no invalidan los resultados, se observa en el análisis global de las dos áreas un incremento en la utilización de antidepresivos acompañado de un cambio en el perfil de prescripción. Desde 1997 hay un claro desplazamiento hacia el consumo de los inhibidores selectivos de la recaptación de serotonina y algunos heterocíclicos (venlafaxina, trazodona y reboxetina), disminuyendo la 
utilización de tricíclicos e IMAO. Esta tendencia era esperable y previsiblemente se seguirá produciendo, ya que los nuevos antidepresivos presentan menos efectos adversos, requieren regímenes posológicos más cómodos, con la supuesta ventaja de menos tasas de abandono ${ }^{21}$ y menor peligro de sobredosificación que los antidepresivos tricíclicos, aún con la consideración de que no existen diferencias significativas de efectividad entre los distintos antidepresivos disponibles. Desde el punto de vista farmacoeconómico el coste directo de los tratamientos con tricíclicos es inferior al que suponen los nuevos medicamentos. En los últimos años, se suceden los estudios que tratan de demostrar que dicha diferencia se ve compensada cuando se consideran los gastos médicos totales y los costes indirectos de los trastornos depresivos. No obstante el patrocinio de estos estudios por parte de las empresas farmacéuticas y su influencia en reuniones científicas y publicaciones difunde un velo de duda acerca de los resultados expuestos ${ }^{22}$.

Un reciente estudio realizado por investigadores de la Universidad de Columbia que analiza la tendencia de tratamiento antidepresivo entre 1987 y 1997, concluye que cada vez son más los estadounidenses tratados por depresión, y que la terapia farmacológica ha experimentado un aumento significativo respecto a la psicoterapia. Al inicio del estudio el $37 \%$ de los pacientes tratados tomaban medicación antidepresiva, proporción que se ve duplicada en 1997 con un $75 \%$. Según los autores, la aparición de la fluoxetina en 1987 es el factor que mayor impacto ha tenido en este contexto23. Similares resultados obtiene el estudio de Alonso et al12 en el que especifica que el consumo de antidepresivos en España se ha incrementado en más de tres veces en la década comprendida entre 1985-1994. Concluye que todos los subgrupos contribuyen en mayor medida al ascenso pero la introducción del los ISRS ha sido determinante. Especifica también que, a pesar del gran incremento experimentado, el consumo de antidepresi- vos se encuentra todavía muy lejos del que podría esperarse teniendo en cuenta los datos de prevalencia real de la depresión, $30 / 1000$ en varones y 40-90/1000 en mujeres.

El aumento que observan estos autores en el incremento de los fármacos antidepresivos continúa produciéndose en las dos áreas de nuestro estudio. En 1997 la DHD en al área de Zamora es de 20,10 y en el área de La Rioja 16,08 , llegando a ser de 34,15 y 31,01 respectivamente en el 2001. El mayor consumo en DHD en el área de Zamora con respecto al área de La Rioja puede ser debido al mayor porcentaje de población pensionista en el área de Zamora con respecto a La Rioja $(37,7 \%$ y $30 \%$ respectivamente del total de la población) y, según datos epidemiológicos, la prevalencia de la depresión es mayor en adultos mayores que en adultos jóvenes. Por otra parte puede haber en el área de Zamora un mayor diagnóstico y tratamiento debido a que el número de profesionales sanitarios que trabajan en Atención Primaria es superior.

El consumo total de antidepresivos tricíclicos disminuye en las dos áreas, con mayor proporción en Zamora respecto a La Rioja, debido a su mayor proporción de población pensionista; ya que aunque son fármacos eficaces presentan un perfil de efectos adversos (anticolinérgicos, cardiotóxicos...) e interacciones más desfavorables que hay que tener en cuenta en personas mayores, ya que en gran parte son pacientes polimedicados, y se han comercializado nuevas moléculas sin estos inconvenientes. Es importante destacar en La Rioja el incremento discreto (8\%) de la amitriptilina, aunque desconocemos a qué puede ser debido.

En cuanto a los IMAO, aunque su eficacia antidepresiva es similar a la del resto de los medicamentos, su utilización se ha visto disminuida considerablemente por la importancia de sus efectos indeseables (posibilidad de crisis hipertensivas con riesgo vital cuan- 
do se producen interacciones con otros medicamentos o con la ingesta de la tiramina contenida en los alimentos), lo que requiere mayor experiencia por parte del médico y una colaboración importante por parte del paciente. Su uso queda restringido a trastornos graves y resistentes.

El grupo de heterocíclicos aumenta considerablemente en el periodo estudiado, a expensas de fármacos de reciente comercialización, como venlafaxina. Este es el cuarto antidepresivo más consumido en Zamora y el sexto en La Rioja, debido al buen perfil de tolerabilidad, eficacia similar a los ISRS, a que se ha sugerido que su efecto antidepresivo puede aparecer de forma más rápida que con el resto de antidepresivos, y también a una fuerte promoción comercial. Venlafaxina se utiliza cada vez más en la clínica diaria como fármaco alternativo a los tricíclicos en depresiones moderadas y graves.

Mianserina disminuye en La Rioja y sin embargo aumenta discretamente en Zamora, debido probablemente a la frecuencia de agranulocitosis asociada al tratamiento. También disminuye maprotilina debido a sus efectos adversos. Ambos tetraciclícos están siendo sustituidos en la practica por ISRS. Aumentan de forma importante, aunque la DHD es mayor en Zamora que en La Rioja: trazodona, mirtazapina, nefazodona (retirada del mercado en marzo del 2003 por presentar un mayor riesgo de producir hepatotoxicidad respecto a otras alternativas terapéuticas). En cuanto a reboxetina; disponemos todavía de pocos datos dada su reciente comercialización (1999). Es un potente inhibidor de la recaptación de noradrenalina con efecto débil sobre la recaptación de serotonina y no afecta a la dopamina. Entre sus efectos adversos se recoge sequedad de boca, estreñimiento y sudoración, efectos que hicieron, entre otras causas, abandonar el consumo de antidepresivos tricíclicos, aunque se ha señalado su posible utilidad en depresiones inhibidas.

En los últimos años estamos asistiendo a un incremento progresivo del consumo de los ISRS respecto al resto de los antidepresivos. Este incremento se debe a que presentan una eficacia similar a la de los tricíclicos sin algunos de sus inconvenientes, lo que mejora la adhesión al tratamiento ${ }^{21}$. El estudio de Baena $^{24}$ señala que es posible que los ISRS en la actualidad no hayan sustituido a los antidepresivos tricíclicos, sino que su extraordinario incremento se deba a un mayor diagnóstico de los trastornos depresivos leves y moderados que anteriormente no recibían tratamiento y que ahora son tratados con fármacos ISRS considerados más seguros y con un perfil de efectos secundarios teóricamente mejor. Las diferencias de consumo entre las dos áreas y entre los distintos principios activos puede explicarse por las diferentes técnicas comerciales ejercidas por los laboratorios, así como por la influencia de los especialistas de referencia ya que los metaanálisis de los ensayos clínicos comparativos de ambas clases de medicamentos muestran una eficacia similar. Debido al perfil de efectos secundarios, aunque la fluvoxamina fue el primer fármaco del grupo comercializado, es el menos utilizado. Sertralina y citalopram presentan el mayor crecimiento dentro del grupo, debido probablemente a su reciente introducción y a que presentan menos interacciones que el resto del grupo, por lo que pueden ser adecuados en pacientes polimedicados, como pueden ser las personas ancianas. La fluoxetina sigue creciendo aunque ha sido desplazada en utilización por paroxetina, debido probablemente a su mayor efecto sedante y a su utilización en trastornos frecuentes como los ansioso depresivos y la fobia social. Paroxetina es el único ISRS indicado por la FDA en el tratamiento de cinco trastornos de ansiedad, además de la depresión.

El importante aumento del diagnóstico de la depresión (según los diferentes estudios) y de las alternativas de tratamiento farmacológico hace preciso consensuar criterios racionales para el uso de fármacos antidepresivos y difundirlos a todos los profesionales médicos. Posteriormente se debería comprobar si 
esta información mejora el diagnóstico y la utilización de estos fármacos en nuestras áreas. Animamos a que se realicen estudios similares en otras áreas que nos permitan comparar nuestros resultados y profundizar en su análisis.

\section{BIBLIOGRAFÍA}

1. Padín JJ, Lozano M, Sabán Ruiz J. Depresión(I). El médico 2001.

2. Celdrán Yelo D, García Domínguez JC, García Marrero $M^{a} R$, López Herrero LS, Llorente San Martín M, Retuerta García D et al. Depresión. En: recomendaciones de la Sociedad Española de Medicina Familiar y Comunitaria. Barcelona: Edide; 2001.

3. Centro Andaluz de Información de Medicamentos. Tratamiento farmacológico de la depresión mayor en Atención Primaria. Granada: Escuela Andaluza de Salud Pública; 1999. Monografía núm 16.

4. Rispau Falgás A, Soler Vila M, García Bayo I, Caramés Durán E, Espín Martínez A, García Pulido C. Factores de riesgo asociados al consumo de antidepresivos. Aten Primaria 1998; 22: 440-3.

5. De la Gándara Martín JJ. Manejo de la depresión y ansiedad en Atención Primaria. Aten Primaria 1997; 20: 389-94.

6. Saiz Ruiz J, Ibáñez Cuadrado A. Tratamiento de los trastornos depresivos. Rev Clin Esp 1997; 197: 3543.

7. La efectividad de los antidepresivos más recetados es similar según un estudio. Jano On-line Febrer (citado 21 de junio 2001). Disponible en: http://db.doyma.es/cgi-bin/wdbcgi.exe/doyma/ press.plantilla?ident $=17352$

8. La depresión afecta a unos 2 millones de españoles. Jano 2002 Febrer (citado 27 de agosto 2001). Disponible en: http://db.doyma.es/cgibin/wdbcgi.exe/doyma/press.plantilla? ident $=15730$.

9. Vázquez-Barquero JL, Díez-Manrique JF, Peña C, Aldama J, Samaniego Rodríguez C, Menéndez Arango J et al. A community mental health survey in Cantabria: a general description of morbidity. Psychol Med 1987; 17: 227-41.

10. Angst J. ¿Cuál es la recurrencia y el pronóstico de la enfermedad depresiva?. En. Montgomery S y
Rouillon F, editores. Tratamiento a largo plazo de la depresión. Chichester: John Wiley Sons; 1992. p.11-23.

11. A systematyc guide for the management of depression in Primary Care. Treatment of depression. CEBMH depression guideline 1998 (citado 18 de diciembre de 1998). Disponible en: http://cebm. warne.ox.ac.uk.

12. Alonso MP, de Abajo FJ, Martínez JJ, Montero D, Martín-Serrano G, Madurga M. Evolución del consumo de antidepresivos en España. Impacto de los inhibidores selectivos de la recaptación de serotonina. Med Clín (Barc) 1997; 108: 161-6.

13. Díaz Madero A, López Ferreras, Ruiz-Clavijo Díez MT, Vargas Aragón ML. Evolución del consumo de antidepresivos en el área de Zamora desde 1996 a 1999. Aten Primaria 2001; 28: 333-9.

14. Boletín Oficial del Estado. Orden Ministerial de 13 de octubre de 1989, por la que se establece la clasificación anatómica de medicamentos oficial de España. BOE núm 257 de 26/10/1989.

15. Antidepresivos. En: Catálogo de especialidades farmacéuticas. Madrid: Consejo General de Colegios Oficiales de Farmacéuticos; 2001.p. 1724-9.

16. Boletín Oficial del Estado. Real Decreto 1663/1998, de 24 de julio, por el que se amplía la relación de medicamentos a efectos de su financiación con cargo a fondos de la Seguridad Social o a fondos estatales afectos a la sanidad. BOE núm 177 de 25/07/1998.

17. Ruiz-Clavijo Diez MT, Sainz de Rozas Aparicio C. Estudio del consumo de antipsicóticos en La Rioja. Años 1997-1998.Impacto de los nuevos antipsicóticos. Pharmaceutical Care 2000; 2(5): 338-46.

18. García Iñesta A. Estudios de utilización de medicamentos en España y análisis de la situación farmacoterapéutica. En: Instituto Nacional de la Salud, editor. Estudios de Utilización de Medicamentos. Madrid: Insalud; 1992.p.15-48.

19. Nomenclator Digitalis. Madrid: Insalud; 2000.

20. World Health Organization. Anatomical therapeutic chemical (ATC) clasification index. Oslo: WHO Collaborating Centre for Drugs Statistics Methodology; 1996.

21. Travé Rodríguez AL, Reneses Sacristán A. Manejo de los fármacos en el tratamiento de la depresión. Información terapéutica del SNS 2002; 26 (1): 1-8.

22. Olfson M, Marcus SC, Druss B, Elinson L, Tanielian T, Pincus HA. National Trends in the Outpa-

Rev Esp Salud Pública 2004, Vol. 78, N. 5 
tient Treatment of Depresión. JAMA 2002; 287 : 203-9.

23. Anderson IM, Nutt DJ, Deakin FW. Evidencebased guidelines for treating depressive disorders with antidepressants: a revision of the 1993 British Association for Psychopharmacology guidelines. J Psychopharmacol 2000; 14(1):3-20.
24. Baena Díez JM, López Mompó C, Fuentes Camps EM, Bragulat Martín A, Cucurull Folguera E, García Hidalgo A et al. Evolución del tratamiento farmacológico de las depresiones(1989-1996): la irresistible ascensión de los inhibidores de la recaptación de serotonina. Medicina familiar y comunitaria 1999; 9(2): 90-7. 